\title{
Significant association between lower pulse pressure and increasing levels of a novel type of phospholipid
}

C.S. Yao ${ }^{1}$, Q.Z. Wu ${ }^{2}$, H.X. Xu ${ }^{3}$, F. Liu ${ }^{4}$, L.G. Chang ${ }^{3}$, L.C. Song ${ }^{3}$, L.S. Gao ${ }^{5}$ and X.L. Meng ${ }^{1}$

${ }^{1}$ Department of Neurology, First Affiliated Hospital of Chinese Army General Hospital, Beijing, China

${ }^{2}$ Department of Neurology, Peking University First Hospital, Beijing, China

${ }^{3}$ Department of Neurology, Third Hospital of Liao-Cheng City,

Shan Dong Province, China

${ }^{4}$ Department of Neurology, People's Hospital of Rui-Cheng County,

Shan Xi Province, China

${ }^{5}$ Department of Internal Medicine, The 8th Hospital of Shi Jia Zhuang City, China

Corresponding author: C.S. Yao

E-mail: cunshanyao@126.com

Genet. Mol. Res. 13 (2): 2922-2930 (2014)

Received May 13, 2013

Accepted August 23, 2013

Published February 21, 2014

DOI http://dx.doi.org/10.4238/2014.February.21.16

\begin{abstract}
The aim of this study was to analyze the association between pulse pressure and a novel type of phospholipid with solubility similar to that of lysophosphatidic acid (LPA), designated as AP, which was reported to be elevated during ischemia. In this cross-sectional study, 416 hypertensive patients and 252 controls aged between 35 and 70 years were enrolled consecutively. Fasting blood samples were extracted for assays of phospholipids and other biomarkers. Compared to controls, the hypertensive patients had higher levels of both LPA [odds ratio $(\mathrm{OR})=3.83$ ] and $\mathrm{AP}(\mathrm{OR}=4.30)$. Changes in blood pressure did not affect the levels of LPA or AP. However AP, but not LPA, levels were significantly higher in patients with lower or higher pulse pressure
\end{abstract}


(Pearson $\chi^{2}=11.239, \mathrm{P}=0.001$ ). For patients whose pulse pressure was $\leq 60 \mathrm{mmHg}$, plasma levels of AP were significantly negatively correlated with pulse pressure. However, this was not observed for LPA and nine other biomarkers, including lipoproteins. Plasma levels of AP increased in hypertensive patients with higher or lower pulse pressure. Thus, attention should be paid to the possibility of cerebral ischemia in hypertensive patients when they have abnormal pulse pressure, especially for those with relatively low pulse pressure.

Key words: Hypertension; Lysophosphatidic acid; Pulse pressure; Ischemia; Novel phospholipid (AP)

\section{INTRODUCTION}

Hypertension is a disease with high prevalence that can cause serious medical conditions, such as cardiovascular events and stroke (both ischemic and hemorrhagic stroke). Controlling patients' blood pressure is one of the most important methods for preventing both cardiovascular and cerebral vascular events (Millar et al., 1999). Compared with systolic pressure and diastolic pressure, pulse pressure is relatively less studied. However, pulse pressure is also a strong risk factor for coronary events (Millar et al., 1999; Millar and Lever, 2000). Some studies have also indicated the significance of pulse pressure in relation to vascular stiffness (Mokhtari et al., 2008; Toprak et al., 2009); however, the biochemical mechanism underlying these relationships remains unclear. In the last two decades, several studies have emerged showing that lysophospholipids play important roles in thrombus formation, atherosclerosis, and so on (Smyth et al., 2008; Pamuklar et al., 2009). Lysophosphatidic acids (LPAs) are released from activated blood platelets, and can be used for future active blood platelets (Gerrard and Robinson, 1989; Eichholtz et al., 1993; Haserück et al., 2004; Khandoga et al., 2008). Siess et al. (1999) found that LPA accumulated in atherosclerotic plaques, and was the primary platelet-activating lipid. The amount of LPA within human carotid atherosclerotic lesions is the highest in the lipid-rich core, the region that is the most thrombogenic and the most prone to rupture (Siess et al., 1999; Rother et al., 2003).

In the course of a previous study on the biological effects of LPA, we found a novel type of phospholipid (abbreviated as "AP") with solubility similar to LPA. This phospholipid was very sensitive to cerebral ischemia (Sun et al., 2002; Yao et al., 2004). Since a lower pulse pressure might be more closely related to impaired hemodynamics and reduced cardiac output (Voors et al., 2005), and LPA was reported to be involved in the etiology of hypertension (Xu et al., 2003), we conducted the present study to investigate the relationship between blood pressure (systolic, diastolic, and pulse pressure) and the levels of LPA and AP.

\section{MATERIAL AND METHODS}

\section{Patient selection}

This cross-sectional study was conducted from March 1, 2007 to December 29, 2008. Written informed consent was obtained from all of the participants. The study protocol was ap- 
proved by the institutional Ethics Committee of the First Affiliated Hospital of Chinese Army General Hospital, where 270 subjects were consecutively admitted in outpatient clinics. The age of subjects ranged from 35 to 70 years, and all had primary hypertension and/or a history of primary hypertension. Some patients had already received medical treatment, including angiotensin-converting enzyme inhibitors, diuretics, and so on. At the time of enrolment, their blood pressures were measured over the brachial artery of the elbow. The same day, LPA, AP, and other clinical diagnostic markers, such as triglyceride (TG), low-density lipoprotein (LDL), total cholesterol (t-CHO), and the like, were assayed. Exclusion criteria included severe liver, kidney, or lung disease, untreated thyroid disease, acute myocardial infarction (within 6 months), acute stroke (within 6 months), hematopathy, acute infections, tumors, severe gynecological diseases, immunization (within 3 months), immunological system diseases, rheumatism, erythematous lupus, allergosis, serious depression, and other severe diseases. For females in their menstruation period, the assays for the levels of LPA and AP were postponed until after their period. Volunteer non-hypertensive subjects were also selected as controls during the same time by the same experienced clinicians. The non-hypertensive subjects were subjected to neurological examinations, and were confirmed to definitely have no hypertension or hypertension history. The exclusion criteria of the controls were similar to those of the cases.

\section{Measurement of blood pressure}

Hypertension was diagnosed according to World Health Organization diagnostic criteria. Blood pressure was measured with a sphygmomanometer. The cuff pressure at which auscultatory (Korotkow) sounds were first heard was defined as the systolic pressure. The diastolic pressure was defined as the cuff pressure at which the Korotkow sounds become muffled. The detailed procedures were as follows: after enrollment, the subjects were told to measure their blood pressure in the two consecutive days between 9:00 and 10:30 am For each day, they were asked to rest for $15 \mathrm{~min}$ before taking the measurement, and then their blood pressure was taken 3 times, with a 5-min interval between each measurement. The final value for blood pressure was defined as the average value of the measurements over the 2 days. Pulse pressure is defined as the difference between the systolic pressure and the diastolic pressure (Eichholtz et al., 1993). We temporarily defined the normal range for pulse pressure to be between 40 and $60 \mathrm{mmHg}$ (Ganong, 1979) because of the lack of consolidated criteria.

\section{Laboratory measurements}

Blood samples were collected in the morning after the participants had fasted for at least $8 \mathrm{~h}$. Plasma lipids, such as TG, LDL, high-density lipoprotein, t-CHO, as well as the prothrombotic marker, D-dimer, blood glucose, and the like were measured routinely by enzymatic methods or immunoassay in the Clinical Laboratory Test Center of the hospital. Details of the assay for AP and LPA are as follows: $4 \mathrm{~mL}$ venous blood was drawn from each participant into commercially available anticoagulant tubes (Beijing TF Co.) in the morning. The lipid extraction reagents were also purchased from Beijing TF Co. (LPA packaged reagents). Whole blood was centrifuged at $8000 \mathrm{~g}$ for $10 \mathrm{~min}$. Then, $1 \mathrm{~mL}$ platelet-poor plasma was obtained from the supernatant. Lipid extraction was performed at $0^{\circ}$ to $4^{\circ} \mathrm{C}$ to minimize damage to ester bonds. The phospholipid extrac- 
tion from plasma was performed according to previously published methods (Kolarovic and Fournier, 1986; Baker et al., 2001), with slight modifications. After the separation of the two phases, we discarded the lower phase. Hydrogen chloride was added to the remaining upper phase to adjust the $\mathrm{pH}$ to 2.0. The lipids were extracted once again as described above. The resulting pooled organic extract was AP. A $50-\mathrm{mL}$ fraction of the pooled organic extract was used to assay for AP. The remaining extracts were dried in vacuo. Each sample was resuspended in $0.3 \mathrm{~mL}$ chloroform:methanol:water: $28 \% \mathrm{NH}_{4} \mathrm{OH}$ $(250: 100: 15: 0.3, \mathrm{v} / \mathrm{v})$, and was immediately filtered through a $3 \mu \mathrm{m}$ Econosphere $50 \mathrm{x}$ $4.6 \mathrm{~mm}$ silica column (Alltech Associates; Deerfield, IL, USA). LPAs were eluted with a mobile phase of chloroform:methanol:water:28\% $\mathrm{NH}_{4} \mathrm{OH}(250: 100: 15: 0.3, \mathrm{v} / \mathrm{v})$ at 0.5 $\mathrm{mL} / \mathrm{min}$. After elution, the LPA and AP concentrations were quantified by measuring the inorganic phosphorus component using colorimetric assays.

\section{Definitions and nomenclature}

The cut-off value for AP was $0.474 \mathrm{mM}$ and it was $3.2 \mu \mathrm{M}$ for LPA. Chronic kidney disease (CKD) was defined as kidney damage for $\geq 3$ months, as defined by structural or functional abnormalities of the kidney, with or without a decreased glomerular filtration rate (GFR). In this study, we only enrolled subjects with stage 3 or lower CKD, that is, GFR $\geq 30$ $\mathrm{mL} / \mathrm{min}$ per $1.73 \mathrm{~m}^{2}$.

\section{Statistical analysis}

Comparisons of data were performed according to the nature of variables. For two samples of continuous variables, the Student $t$-test was used, whereas for categorical variables, the chi-squared test was used. Bivariate linear correlation analysis and binary logistic regression analysis were performed to investigate the relationship of AP with pulse pressure and other factors. All statistical analyses were performed with the SPSS software package for Windows version 13.0. The statistical significance level was set at $\mathrm{P}<0.05$.

\section{RESULTS}

The baseline characteristics of hypertensive patients and non-hypertensive subjects are shown in Table 1. Clinical and laboratory data, including age, gender, diabetes mellitus, atherosclerosis, hypercholesterolemia, and smoking status, were not significantly different between these two groups. None of the controls had high blood pressure or histories of hypertension.

Correlation analysis between LPA and some risk factors in hypertensive patients was conducted. The Pearson correlation coefficients were: age, $\mathrm{r}=0.063, \mathrm{P}=0.200$; diabetes history, $\mathrm{r}=0.010, \mathrm{P}=0.839$; LDL, $\mathrm{r}=0.091, \mathrm{P}=0.064$; tCHO, $\mathrm{r}=0.131, \mathrm{P}=0.008 ; \mathrm{TG}, \mathrm{r}=$ $0.085, \mathrm{P}=0.083$; smoking history, $\mathrm{r}=-0.132, \mathrm{P}=0.008$; $\mathrm{CKD}, \mathrm{r}=0.073, \mathrm{P}=0.137$.

Hypertensive patients had higher levels of AP and LPA than controls. Table 2 shows the comparison results including the odds ratios (ORs) with 95\% confidence intervals (CI). The number of individuals with AP $\geq 0.474 \mathrm{mM}$ (cut-off value) was significantly higher in the hypertensive patient group than in the control group. The mean value of AP was also significantly larger in the patient group. The results of LPA were similar to those of AP. 
Table 1. Baseline characteristics of hypertensive patients and controls.

\begin{tabular}{|c|c|c|c|c|}
\hline & Hypertensive patients & Controls & Pearson $\chi^{2}$ & $\mathrm{P}$ \\
\hline $\mathrm{N}$ & 416 & 252 & & \\
\hline \multicolumn{5}{|l|}{ Demographic } \\
\hline Age (mean $\pm \mathrm{SD}$, years) & $66.1 \pm 12.9$ & $64.8 \pm 15.8$ & & $0.247 *$ \\
\hline Male & $201(48.3 \%)$ & $132(52.4 \%)$ & 1.037 & 0.309 \\
\hline \multicolumn{5}{|l|}{ Risk factors } \\
\hline Diabetes mellitus & $43(10.3 \%)$ & $17(6.9 \%)$ & 2.905 & 0.116 \\
\hline Atherosclerosis** & $59(14.1 \%)$ & $29(11.6 \%)$ & 0.982 & 0.322 \\
\hline Smoking & $65(15.7 \%)$ & $33(12.9 \%)$ & 0.802 & 0.370 \\
\hline Hypercholesterolemia & $57(13.6 \%)$ & $23(9.1 \%)$ & 3.116 & 0.078 \\
\hline Chronic kidney diseases & $30(7.2 \%)$ & $7(2.8 \%)$ & 5.896 & 0.015 \\
\hline Hypertension $^{\ddagger}$ & $416(100 \%)$ & $0(0 \%)$ & & \\
\hline Treated by ACEIs or ARBs & $140(33.7 \%)$ & & & \\
\hline Treated by $\beta$-blockers & $99(23.7 \%)$ & & & \\
\hline Treated by CCBs & $119(28.5 \%)$ & & & \\
\hline Treated by diuretics & $72(17.3 \%)$ & & & \\
\hline
\end{tabular}

Data are reported as number with percent in parentheses. *Student $t$-test is used, $\mathrm{t}=1.158$. Values are compared by

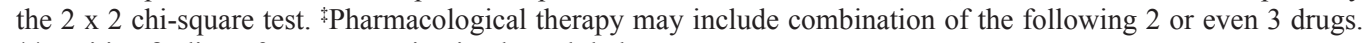
**Positive finding of artery examination by ophthalmoscope.

Table 2. Hypertensive patients have elevated levels of both AP and LPA Comparing with controls.

\begin{tabular}{|c|c|c|c|c|}
\hline & Hypertensive patients & Controls & $\mathrm{P}$ & OR $(95 \% \mathrm{CI})$ \\
\hline \multicolumn{5}{|l|}{ Comparison of AP } \\
\hline $\mathrm{AP}<0.474 \mathrm{mM}$ & $194(46.6 \%)$ & $199(79.0 \%)$ & & \\
\hline $\mathrm{AP} \geq 0.474 \mathrm{mM}$ & $222(53.4 \%)$ & $53(21.0 \%)$ & & \\
\hline Total & 416 & 252 & $0.00^{\dagger}$ & $4.30(3.00-6.15)$ \\
\hline $\mathrm{AP}($ means $\pm \mathrm{SD} \mathrm{mM})$ & $0.550 \pm 0.211$ & $0.387 \pm 0.158$ & $0.00(\mathrm{t}=10.59)^{\ddagger}$ & \\
\hline \multicolumn{5}{|l|}{ Comparison of LPA } \\
\hline $\mathrm{LPA}<3.2 \mu \mathrm{M}$ & $185(44.4 \%)$ & $190(75.5 \%)$ & & \\
\hline $\mathrm{LPA} \geq 3.2 \mu \mathrm{M}$ & $231(55.6 \%)$ & $62(24.5 \%)$ & & \\
\hline Total & 416 & 252 & $0.00^{\S}$ & $3.83(2.71-5.41)$ \\
\hline $\mathrm{LPA}($ means $\pm \mathrm{SD} \mu \mathrm{M})$ & $4.04 \pm 2.81$ & $2.82 \pm 1.83$ & $0.00(\mathrm{t}=6.15)^{\ddagger}$ & \\
\hline
\end{tabular}

Data are reported as number with percent in parentheses, unless otherwise indicated. ${ }^{\dagger}$ Compared by the $2 \times 2$ chisquare test. $\chi^{2}=67.74$. ${ }^{\star}$ Compared by Student $t$-test. ${ }^{\circledR}$ Compared by the $2 \times 2$ chi-square test. $\chi^{2}=60.96$.

Although hypertensive patients had higher levels of both phospholipids, the status of blood pressure measured during the study did not significantly affect the levels of LPA or AP. We divided the patients into 4 groups: 1 ) normal blood pressure (note: hypertension patients had been treated with medicine); 2) only systolic blood pressure elevated;3) only diastolic pressure elevated; and 4) both systolic and diastolic pressure elevated. The results of the 4 $\mathrm{x} 2$ chi-squared test and one-way analysis of variance (ANOVA) showed that there were no significant differences between any groups (Table 3 ).

Although the status of blood pressure did not affect the levels of LPA or AP, this was not the case for pulse pressure. We divided the patients into two groups: group 1 had a medium range ( $40 \leq \mathrm{PP}<60 \mathrm{mmHg}$ ) of pulse pressure and group 2 had either higher or lower pulse pressure ( $\mathrm{PP}<40$ or $\mathrm{PP} \geq 60$ ). The $2 \times 2$ chi-squared test results showed that the AP level in group 1 was significantly lower than that of group 2 (Table 4). However, for LPA, this difference was not significant (data not shown). The results of the $3 \times 2$ chi-squared test for the three categories of pulse pressure ( $\mathrm{PP}<40,40 \leq \mathrm{PP}<60$, and $\mathrm{PP} \geq 60 \mathrm{mmHg}$ ) showed a significant influence of plasma $\mathrm{AP}\left(\chi^{2}=10.775, \mathrm{P}=0.005\right)$. 
Table 3. Comparisons of plasma AP and LPA for hypertensive patients according to different categories of blood pressure.

\begin{tabular}{lcccc}
\hline & Normal BP & SP elevated & DP elevated & Both elevated \\
\hline Comparison of AP* & & & & \\
$\mathrm{AP}<0.474 \mathrm{mM}$ & $53(52.0 \%)$ & $70(44.3 \%)$ & $4(23.5 \%)$ & $64(46.0 \%)$ \\
$\mathrm{AP} \geq 0.474 \mathrm{mM}$ & $49(48.0 \%)$ & $88(55.7 \%)$ & $13(76.5 \%)$ & $75(54.0 \%)$ \\
Total & 102 & 158 & 17 & 139 \\
AP $($ means \pm SD mM) & $0.512 \pm 0.298$ & $0.551 \pm 0.293$ & $0.602 \pm 0.316$ & $0.525 \pm 0.325$ \\
Comparison of LPA & $50(49 \%)$ & $68(43.0 \%)$ & $7(41.2 \%)$ & $67(48.2 \%)$ \\
LPA $<3.2 \mu \mathrm{M}$ & $52(51.0 \%)$ & $90(57.0 \%)$ & $10(58.8 \%)$ & $72(51.8 \%)$ \\
LPA $\geq 3.2 \mu \mathrm{M}$ & 102 & 158 & 17 & 139 \\
Total & $3.89 \pm 1.88$ & $4.28 \pm 2.57$ & $4.44 \pm 2.79$ & $4.47 \pm 2.05$ \\
LPA (means $\pm \mathrm{SD} \mu \mathrm{M})$ & & & \\
\hline
\end{tabular}

Data are reported as numbers with percent in parentheses unless otherwise indicated. $\mathrm{BP}=$ blood pressure; $\mathrm{SP}=$ systolic pressure; DP = diastolic pressure. *AP: comparison of Pearson numbers by the $2 \times 4$ chi-square, Pearson $\chi^{2}$ $=5.098, \mathrm{P}=0.165$; comparison of average values of $\mathrm{AP}$ among groups by one-way ANOVA $\mathrm{F}=2.307 ; \mathrm{P}=0.076$. 'LPA: 2 x 4 chi-square test: Pearson $\chi^{2}=1.358, \mathrm{P}=0.715$; comparison of average values of LPA among groups by one-way ANOVA $\mathrm{F}=1.403 ; \mathrm{P}=0.241$.

Table 4. Influence of different categories of pulse pressure (PP) on plasma AP.

\begin{tabular}{lccc}
\hline & $40 \leq \mathrm{PP}<60 \mathrm{mmHg}$ & $\mathrm{PP}<40$ or PP $\geq 60 \mathrm{mmHg}$ & OR $(95 \% \mathrm{CI})$ \\
\hline Comparison of number & $90(54.5 \%)$ & $95(37.8 \%)$ & \\
$\mathrm{AP}<0.474 \mathrm{mM}$ & $75(45.5 \%)$ & $156(62.2 \%)$ & $1.971(1.323-2.936)$ \\
$\mathrm{AP} \geq 0.474 \mathrm{mM}$ & 165 & 251 & $0.001^{*}$ \\
Total & & & \\
Comparison of concentration & $0.464 \pm 0.192$ & $0.571 \pm 0.218$ & $0.000^{\dagger}$ \\
AP (means \pm SD mM) & $3.65 \pm 2.26$ & $3.87 \pm 2.37$ & $0.346^{\ddagger}$ \\
LPA (means \pm SD $\mu \mathrm{M})$ &
\end{tabular}

*Chi-square test, Pearson $\chi^{2}=11.239 .{ }^{\dagger}$ Student $t$-test $(\mathrm{t}=5.131) .{ }^{\star}$ Student $t$-test $(\mathrm{t}=0.943)$.

Bivariate linear correlation analysis showed that plasma AP levels were significantly negatively correlated with pulse pressure when PP $\geq 60 \mathrm{mmHg}$. However, LPA and the other 9 other widely used clinical markers, such as lipoproteins, glucose, and so on, were not significantly correlated with pulse pressure (Table 5).

Table 5. Correlation coefficient for the relationship between age and each of the biomarkers with pulse pressure.

\begin{tabular}{lccc}
\hline Marker & $\mathrm{N}$ & Pearson's correlation coefficient & P \\
\hline Age $($ years $)$ & 416 & 0.167 & $0.000^{*}$ \\
AP $(\mathrm{mM})$ & 416 & -0.208 & $0.000^{*}$ \\
LPA $(\mu \mathrm{M})$ & 416 & -0.070 & 0.154 \\
t-CHO $(\mathrm{mM})$ & 404 & -0.031 & 0.534 \\
HDL $(\mathrm{mM})$ & 404 & -0.076 & 0.127 \\
LDL $(\mathrm{mM})$ & 404 & 0.081 & 0.104 \\
TG $(\mathrm{mM})$ & 404 & -0.025 & 0.616 \\
Glucose $(\mathrm{mM})$ & 399 & 0.028 & 0.577 \\
Lipoprotein $(\mathrm{a})(\mathrm{g} / \mathrm{L})$ & 393 & -0.065 & 0.220 \\
Lipoprotein $(\mathrm{b})(\mathrm{g} / \mathrm{L})$ & 390 & 0.061 & 0.229 \\
D-dimer $(\mu \mathrm{g} / \mathrm{L})$ & 377 & -0.071 & 0.169 \\
Uric acid $(\mu \mathrm{M})$ & 367 & 0.066 & 0.207 \\
\hline
\end{tabular}

The correlation analysis was performed for hypertensive patients with pulse pressure $\leq 60 \mathrm{mmHg}$. ${ }^{*}$ Correlation statistically significant. 
To compare the effects of blood pressure and pulse pressure on AP, multivariate binary logistic regression was performed using the step-by-step backward method, with the probabilities for the stepwise method set to an entry level of $\mathrm{P}=0.05$ and a removal level of $\mathrm{P}=$ 0.1 . The results showed that both systolic pressure and diastolic pressure were disregarded from further statistical analysis, and only pulse pressure was finally included in the regression equation: $\log \mathrm{P}=-0.399+0.859$ pulse pressure, where $\mathrm{P}$ is the probability of AP elevation, and pulse pressure is defined as a binary categorical variable with a value of either $0(40 \leq \mathrm{PP}$ $<60)$ or $1(\mathrm{PP}<40$ or $\mathrm{PP} \geq 60)$.

This result indicated that only pulse pressure significantly influences the AP plasma level. Results of the chi-squared test showed that the logistic regression equation was statistically significant $\left(\chi^{2}=5.236\right.$ and $\left.\mathrm{P}=0.022\right)$.

\section{DISCUSSION}

Although primary hypertension is a very common disease, its etiology is still not entirely clear. The relationship among systolic pressure, diastolic pressure, pulse pressure, and vascular events are relatively complex. However, according to the results of studies conducted to date, we can almost undoubtedly conclude that hypertension is one of the most important risk factors of vascular events. Elucidating the underlying mechanism will involve the combined study of basic medical science and clinical studies, including the study of new surrogate markers (Cohn, 2004). The LPA family has recently been recognized as important phospholipids with strong thrombogenic effects. In particular, AP is a promising surrogate marker for ischemia. The pathological production of AP theoretically involves the following steps. First, reactive oxygen species increase when the organ is in ischemia (Sowers, 2002). Then, the oxidative stress enhances the activities of phospholipase D and phospholipase A2 (Goto et al., 1988; Ito et al., 1997; Banno et al., 2001; Min et al., 2007). This activated enzyme catalyzes the breakdown of the chemical bonds of phospholipid molecules, and eventually produces AP.

Our current study shows that patients with either higher or lower pulse pressure have increased levels of AP. Petrie et al. (2009) proposed that pulse pressure is a reflection of the atherosclerotic burden on the one hand (high pulse pressure), and left ventricular dysfunction on the other hand (low pulse pressure), and that these two entities may counteract each other. Our result is consistent with this report. Furthermore, our results showed that there was a significantly negative correlation between AP and lower or higher pulse pressure. Based on the nature of AP described above, this result indicates that patients with a low pulse pressure would be more prone to ischemia. This deduction is based on current understanding of the nature of pulse pressure. Theoretically speaking, pulse pressure is positively proportional to heart stroke output, and is negatively proportional to aorta compliance. Therefore, a lower pulse pressure reflects a lower heart output when both the heartbeat and aorta compliance are relatively constant. Petrie et al. (2009) also proposed that pulse pressure might be closely related to stroke volume, and that low pulse pressure probably reflects decreased cardiac function (Voors et al., 2005). As a result of decreased heart stroke output accompanied by low pulse pressure, some organs, such as the brain, might ultimately manifest ischemia. Our current study showed that ischemia could be indicated by an elevated AP plasma level.

There is no clearly defined normal range of pulse pressure for clinical practice, despite the fact that there is ample evidence suggesting that pulse pressure plays an important role, at 
least in cardiovascular events (Benetos et al., 1997; Verdecchia et al., 1998; Franklin et al., 1999). Voors et al. (2005) adopted $45 \mathrm{mmHg}$ as the median value of pulse pressure. For our study, we temporarily defined the normal range of pulse pressure to be between 40 and $60 \mathrm{mmHg}$ (Ganong, 1979); however, it should be noted that according to the Review of Medical Physiology, pulse pressure is normally about $50 \mathrm{mmHg}$. Therefore, this definition should be verified further.

AP levels varied among patients with either higher or lower pulse pressure. Physicians should pay attention to the possibility of (cerebral) ischemia in hypertensive patients with higher or especially lower pulse pressure. AP is a very promising marker for ischemic vascular diseases.

\title{
ACKNOWLEDGMENTS
}

\author{
Research supported by a grant of the First Affiliated Hospital of Chinese Army General \\ Hospital (\#YJ2009330).
}

\section{Conflicts of interest}

The authors declare no conflict of interest.

\section{REFERENCES}

Baker DL, Desiderio DM, Miller DD, Tolley B, et al. (2001). Direct quantitative analysis of lysophosphatidic acid molecular species by stable isotope dilution electrospray ionization liquid chromatography-mass spectrometry. Anal. Biochem. 292: 287-295.

Banno Y, Wang S, Ito Y, Izumi T, et al. (2001). Involvement of ERK and p38 MAP kinase in oxidative stress-induced phospholipase D activation in PC12 cells. Neuroreport 12: 2271-2275.

Benetos A, Safar M, Rudnichi A, Smulyan H, et al. (1997). Pulse pressure: a predictor of long-term cardiovascular mortality in a French male population. Hypertension 30: 1410-1415.

Cohn JN (2004). Introduction to surrogate markers. Circulation 109: IV20-IV21.

Eichholtz T, Jalink K, Fahrenfort I and Moolenaar WH (1993). The bioactive phospholipid lysophosphatidic acid is released from activated platelets. Biochem. J. 291 (Pt 3): 677-680.

Franklin SS, Khan SA, Wong ND, Larson MG, et al. (1999). Is pulse pressure useful in predicting risk for coronary heart Disease? The Framingham heart study. Circulation 100: 354-360.

Ganong WF (1979). Review of Medical Physiology. 9th edn. Lange Medical Publications, Norwalk.

Gerrard JM and Robinson P (1989). Identification of the molecular species of lysophosphatidic acid produced when platelets are stimulated by thrombin. Biochim. Biophys. Acta 1001: 282-285.

Goto Y, Okamoto S, Yonekawa Y, Taki W, et al. (1988). Degradation of phospholipid molecular species during experimental cerebral ischemia in rats. Stroke 19: 728-735.

Haserück N, Erl W, Pandey D, Tigyi G, et al. (2004). The plaque lipid lysophosphatidic acid stimulates platelet activation and platelet-monocyte aggregate formation in whole blood: involvement of P2Y1 and P2Y12 receptors. Blood 103: 2585-2592.

Ito Y, Nakashima S and Nozawa Y (1997). Hydrogen peroxide-induced phospholipase D activation in rat pheochromocytoma PC12 cells: possible involvement of $\mathrm{Ca}^{2+}$-dependent protein tyrosine kinase. J. Neurochem. 69: 729-736.

Khandoga AL, Fujiwara Y, Goyal P, Pandey D, et al. (2008). Lysophosphatidic acid-induced platelet shape change revealed through LPA(1-5) receptor-selective probes and albumin. Platelets 19: 415-427.

Kolarovic L and Fournier NC (1986). A comparison of extraction methods for the isolation of phospholipids from biological sources. Anal. Biochem. 156: 244-250.

Millar JA and Lever AF (2000). Implications of pulse pressure as a predictor of cardiac risk in patients with hypertension. Hypertension 36: 907-911.

Millar JA, Lever AF and Burke V (1999). Pulse pressure as a risk factor for cardiovascular events in the MRC Mild Hypertension Trial. J. Hypertens. 17: 1065-1072. 
Min dS, Choi JS, Kim HY, Shin MK, et al. (2007). Ischemic preconditioning upregulates expression of phospholipase D2 in the rat hippocampus. Acta Neuropathol. 114: 157-162.

Mokhtari A, Bellinetto-Ford L, Melander O and Nilsson PM (2008). Determinants of increasing pulse pressure during 23 years' follow-up as a marker of arterial stiffness and vascular ageing. Blood Press 17: 291-297.

Pamuklar Z, Federico L, Liu S, Umezu-Goto M, et al. (2009). Autotaxin/lysopholipase D and lysophosphatidic acid regulate murine hemostasis and thrombosis. J. Biol. Chem. 284: 7385-7394.

Petrie CJ, Voors AA and van Veldhuisen DJ (2009). Low pulse pressure is an independent predictor of mortality and morbidity in non ischaemic, but not in ischaemic advanced heart failure patients. Int. J. Cardiol. 131: 336-344.

Rother E, Brandl R, Baker DL, Goyal P, et al. (2003). Subtype-selective antagonists of lysophosphatidic acid receptors inhibit platelet activation triggered by the lipid core of atherosclerotic plaques. Circulation 108: 741-747.

Siess W, Zangl KJ, Essler M, Bauer M, et al. (1999). Lysophosphatidic acid mediates the rapid activation of platelets and endothelial cells by mildly oxidized low density lipoprotein and accumulates in human atherosclerotic lesions. Proc. Natl. Acad. Sci. U. S. A. 96: 6931-6936.

Smyth SS, Cheng HY, Miriyala S, Panchatcharam M, et al. (2008). Roles of lysophosphatidic acid in cardiovascular physiology and disease. Biochim. Biophys. Acta 1781: 563-570.

Sowers JR (2002). Hypertension, angiotensin II, and oxidative stress. N. Engl. J. Med. 346: 1999-2001.

Sun YH, Wu QZ and Yao CS (2002). A preliminary study of plasma lysophosphatidic acid levels in patients with ischemic stroke. Chin. J. Med. 82: 229-231.

Toprak A, Reddy J, Chen W, Srinivasan S, et al. (2009). Relation of pulse pressure and arterial stiffness to concentric left ventricular hypertrophy in young men (from the Bogalusa Heart Study). Am. J. Cardiol. 103: 978-984.

Verdecchia P, Schillaci G, Borgioni C, Ciucci A, et al. (1998). Ambulatory pulse pressure: a potent predictor of total cardiovascular risk in hypertension. Hypertension 32: 983-988.

Voors AA, Petrie CJ, Petrie MC, Charlesworth A, et al. (2005). Low pulse pressure is independently related to elevated natriuretic peptides and increased mortality in advanced chronic heart failure. Eur. Heart J. 26: 1759-1764.

Xu YJ, Aziz OA, Bhugra P, Arneja AS, et al. (2003). Potential role of lysophosphatidic acid in hypertension and atherosclerosis. Can. J. Cardiol. 19: 1525-1536.

Yao CS, Meng XL and Wu QZ (2004). The worth of lysophosphatidic acid in evaluation of treatment effectiveness and prognosis of early stage of ischemic cerebral vascular diseases. Chin. J. Clinical Rehabilitation 8: 710-711. 